\title{
APOE E4 Carriers Show Prospective Memory Enhancement Under Nicotine, and Evidence for Specialisation Within Medial BAIO
}

\author{
Simon Evans*,', Marcus A Gray ${ }^{2,3}$, Nicholas G Dowell ${ }^{4}$, Naji Tabet ${ }^{5}$, Paul S Tofts ${ }^{4}$, Sarah L King' and \\ Jennifer M Rusted' \\ 'School of Psychology, University of Sussex, Brighton, UK; ${ }^{2}$ Centre for Advanced Imaging, Gehrmann Laboratory, The University of Queensland, \\ St Lucia, Queensland; ${ }^{3}$ University of Queensland Centre for Clinical Research, Royal Brisbane Women's Hospital, Herston, Queensland; ${ }^{4}$ Brighton \\ and Sussex Medical School (BSMS), Clinical Imaging Sciences Centre, Brighton, UK; ${ }^{5}$ Brighton and Sussex Medical School, Institute of \\ Postgraduate Medicine, Brighton, UK
}

\begin{abstract}
There is evidence to suggest that the APOE $\varepsilon 4$ allele (which confers an increased risk of developing dementia) might be associated with cognitive advantages earlier in life. Further, nicotine might selectively benefit $\varepsilon 4$ carriers. We used fMRI to explore performance on a prospective memory (PM) task in young adults (age 18-30) with and without nicotine using a within-subjects design. Participants performed an ongoing task while retaining a PM instruction to respond to specific stimuli embedded in the task. Nicotine effects varied according to APOE status. Reaction times to the PM cue were improved under nicotine in $\varepsilon 4$ carriers, but not in $\varepsilon 3$ carriers. In an eventrelated analysis, extrastriate responses to PM trials were enhanced by nicotine only in $\varepsilon 4$ carriers. These differences in early visual processing may contribute to the behavioral findings. Activity in medial BAIO (previously implicated in PM) differentiated $\varepsilon 4$ from $\varepsilon 3$ carriers. One BAIO subregion showed greater deactivation in $\varepsilon 4$ carriers during PM trials. Activity in other BAIO subregions was modulated by PM reaction time, pointing to region-specific effects within medial BA I0. In addition, activity in right hippocampal formation was only seen in $\varepsilon 4$ carriers receiving nicotine. These results demonstrate that cognitive enhancement by nicotine can selectively benefit $\mathrm{APOE} \varepsilon 4$ carriers, and point to genotype-specific differences in neural activity during PM. In addition, these results show that the role of medial BA 10 in PM likely involves varying contributions from functionally specific subregions.

Neuropsychopharmacology (20I2) 38, 655-663; doi:I0.1038/npp.20I2.230; published online 12 December 2012
\end{abstract}

Keywords: Alzheimer's disease; psychopharmacology; imaging; learning and memory; nicotine; APOE

\section{INTRODUCTION}

Apolipoprotein E (APOE) is a polymorphic protein implicated in mechanisms of neurogenesis, plasticity, and repair (Mahley, 1988). In humans, the APOE gene has three allelic variants $(\varepsilon 2, \varepsilon 3$, and $\varepsilon 4)$. The $\varepsilon 4$ allele is a wellestablished genetic risk factor for Alzheimer's disease (AD) (Rocchi et al, 2003), it is also associated with poorer recovery from brain trauma (Zhou et al, 2008), and an increased incidence of age-related cognitive impairment (Deary et al, 2002).

However, and somewhat paradoxically, there is some evidence that the $\varepsilon 4$ allele may be cognitively advantageous in early life. It has been reported that young $\varepsilon 4$ carriers are more likely to progress to higher education (Hubacek et al, 2001), and at university age have higher IQs (Yu et al, 2000).

*Correspondence: Dr Simon Evans, School of Psychology, University of Sussex, Falmer, Brighton, East Sussex BNI 9QG, UK, Tel: +44 01273 876776, Fax: +44 0I273 678058, E-mail: sle27@sussex.ac.uk

Received 5 July 2012; revised 18 October 2012; accepted 19 October 2012; accepted article preview online 6 November 2012
In this age group, $\varepsilon 4$ carriers also seem to outperform their peers on certain cognitive measures including those of episodic memory (Mondadori et al, 2007) and verbal fluency (Marchant et al, 2010). It has been suggested that this association is an example of antagonistic pleiotropy (Han and Bondi, 2008), where a gene impacts fitness differently across the lifespan. Studies in young adults suggest that, relative to their $\varepsilon 3$ peers, $\varepsilon 4$ carriers show performance-related decreases in neural activity during learning, suggesting enhanced neural efficiency (Mondadori et al, 2007). Some recent structural imaging work supports this argument (Ruest et al (under review)). However, older, healthy, $\varepsilon 4$ carriers show overactivity in task-relevant brain areas during learning and memory tasks (Bondi et al, 2005; Bookheimer et al, 2000; Han et al, 2007) supporting an alternative, 'compensatory' hypothesis where the $\varepsilon 4$ polymorphism confers a disadvantage from the outset, and induced compensatory overactivations drive the cognitive benefits in younger age groups.

Neuroimaging studies have begun to characterize differences in the way $\varepsilon 4$ carriers recruit specific brain regions, both during tasks and in the resting state. At rest, $\varepsilon 4$ carriers 
show co-activation differences within the default mode network (DMN) (Filippini et al, 2009; Westlye et al, 2011). Task-based imaging studies have tended to use episodic memory paradigms to focus on the relationship between hippocampal function and APOE status, since the hippocampus is one of the first regions to be affected by AD (Braak and Braak, 1997) and hippocampal functional differences may therefore be indicative of $\mathrm{AD}$ risk. However, studies have reported both increased (Han et al, 2007) and decreased (Borghesani et al, 2008) activity in the medial temporal lobe (MTL) in $\varepsilon 4$ carriers, albeit using different tasks. These differences occur in the absence of behavioral differences between groups. Age appears to be factor, with $\varepsilon 4$ carriers showing a disproportionate decrease in MTL activity over the lifespan (Filippini et al, 2011). Inconsistent results might also be due to the baseline condition employed, since there is evidence that resting state activity in hippocampal networks might be altered by E4 status (Trachtenberg et al, 2012b), thus complicating event-related designs.

Our aim in the current work was to contrast performance in young adult $\varepsilon 3$ and $\varepsilon 4$ carriers on a measure of prospective memory (PM). PM requires participants to interrupt an ongoing activity to recall and act on an earlier formed intention. Successful PM function is critical to living independently in the community and is thus a useful index of real-world memory performance. Possession of the $\varepsilon 4$ allele has been shown to negatively impact PM performance in normally aging older adults (Driscoll et al, 2005). However, in young adults, it has been reported that the $\varepsilon 4$ allele confers an advantage in PM task performance (Marchant et al, 2010). Thus, PM tasks usefully illustrate the antagonistic pleiotropy concept. Furthermore, while the cholinergic agonist nicotine has been shown to enhance PM performance (Marchant et al, 2008; Rusted et al, 2009, 2005), improvements were more significant in $\varepsilon 4$ carriers (Marchant et al, 2010). The $\varepsilon 4$ allele has been associated with deficient cholinergic activity (Arendt et al, 1997; Poirier et al, 1995), which may explain why $\varepsilon 4$ carriers could be more advantaged by nicotine. In this study, we focused on PM performance in young adults, with each participant tested after receiving nicotine or placebo in a double-blind, randomized, crossover design. PM tasks typically activate frontal and parietal regions (Burgess et al, 2011). Previous work with this task found that PM trials increased activity within inferior parietal, dorsal ACC and lateral prefrontal regions, and decreased medial prefrontal activity. Details of the network activated by PM trials in this task can be found in Rusted et al (2011). Based on these previous behavioral findings, we expected nicotine to selectively benefit $\varepsilon 4$ carriers. In an event-related design, we explored which brain structures might mediate this effect. We also examined genotype differences in task-related activity under placebo. In contrast to many other PM studies using blocked designs, our event-related design allowed us to localize activity modulated by reaction time, on a trial-by-trial basis. This not only allowed us to determine which regions are most closely implicated in PM performance, but could also be used to show whether possible compensatory overactivations in $\varepsilon 4$ carriers are beneficial in this task context.

\section{MATERIALS AND METHODS}

\section{Participants}

Ninety-eight healthy participants (aged 18-30 years) were recruited from the University of Sussex by means of e-mail and through the university online recruitment program. All participants were right-handed Caucasian undergraduates at the University of Sussex. Volunteers were excluded from the study for untreated high blood pressure or heart problems, a history of psychiatric or neurological illness, including the use of psychoactive medication. Also excluded were pregnant mothers and those with metallic implants including bridges and braces, or tattoos above the shoulder. All participants were non-smokers (and had been for at least 5 years), had a body mass index within the normal range and had English as their first language.

APOE genotype was determined by buccal swab. APOE genotypes were determined by KBiosciences (Hoddesdon, UK; www.kbioscience.co.uk), using their own system of fluorescence-based competitive allele-specific PCR (KASPar). Two APOE single-nucleotide polymorphisms (SNPs) rs429358 and rs7412 allowed identification of the three major APOE alleles $(\varepsilon 2, \varepsilon 3$, and $\varepsilon 4)$. In line with evidence suggesting the $\varepsilon 2$ allele may influence cognition (Bloss et al, 2010), volunteers heterozygous for a single $\varepsilon 2$ allele were excluded. From the 98 volunteers, 40 volunteers were selected for further study of which 20 were either homozygous or heterozygous for the $\varepsilon 4$ allele (henceforth referred to as $\varepsilon 4$ carriers) and 20 of which were homozygous for the $\varepsilon 3$ allele (henceforth referred to as 83 carriers). Among the $\varepsilon 4$ carriers, two participants were homozygous e4 carriers.

Volunteers were all aged between 19 and 24 years $(\mathrm{M}=20.2$ years, $\mathrm{SD}=1.9), 14$ male and 26 female. Further demographic information can be found in Table 1. There was no difference in gender, age, or baseline cognitive performance between groups.

\section{Experimental Design}

All participants volunteered under a written informed consent procedure approved by the Sussex University

Table I Volunteer Characteristics and Measures of Baseline Cognitive Performance.

\begin{tabular}{lcccc}
\hline \multicolumn{1}{c}{ Group } & Age & Gender & NART IQ & Episodic memory (words recalled/20) \\
\hline $\mathrm{e} 4+(n=20)$ & $21.5 \pm 2.3$ & $12 \mathrm{~F}$ & $114 \pm 4$ & $9.2 \pm 2.7$ \\
$\mathrm{e} 4-(n=20)$ & $20.9 \pm 1.4$ & $14 \mathrm{~F}$ & $115 \pm 3$ & $9.7 \pm 2.9$ \\
Sig. test $(P$-value $)$ & 0.41 & 0.52 & 0.29 & 0.50 \\
\hline
\end{tabular}

Groups did not differ on age, gender, or baseline cognitive measures. 
Schools of Psychology and Life Sciences Research Ethics Committee. Participants attended three sessions. In an initial session, participants were tested on baseline measures of cognitive performance. IQ was measured using the National Adult Reading Test (NART; Nelson, 1982). Episodic memory was measured through immediate free recall of a 20 -item word list, presented at a rate of 1 word every $2 \mathrm{~s}$ (procedures and materials from Rusted and Warburton, 1989). Participants were also familiarized with the card sort task, and practised self-administering the nasal spray. In each of the subsequent scanning sessions, volunteers received a short practice run with the ongoing card sort task without PM instruction (see below). Nasal sprays (placebo or nicotine) were self-administered using double-blind procedures, with fMRI scanning commencing 18-20 min later. In the first scanning session, half of the participants received nicotine and the other half placebo, with the alternative treatment received in the second session. All three sessions were spaced $\sim 1$ week apart. The two session within-subject manipulation used here is essential to the pharmacological/neuroimaging component of this study, which cannot be completed in a single session. Although a departure from a standard PM paradigm, our previous work has shown that the PM intention effect is sustained across sessions, despite lack of naïvity (Rusted et al, 2011).

\section{Nicotine Delivery}

Nasal sprays comprised individual bottles with mechanical spray pumps that delivered $0.5 \mathrm{mg}$ of nicotine or a perceptually matched inactive placebo per spray. Nasal sprays were coded by an independent third party and each volunteer was allocated to receive nicotine in one session and placebo in the other, with the order counterbalanced across participants. For each session, volunteers self-administered two puffs from the allocated spray, one in each nostril, delivering $1.0 \mathrm{mg}$ nicotine or sensory-matched placebo. Peak plasma levels are reached $\sim 15$ min after delivery (Schneider et al, 1996); halflife range for nicotine is $1-2 \mathrm{~h}$. Nasal sprays were provided by AB McNeil, Helsingborg, Sweden.

\section{PM Task}

The ongoing task involved simply sorting computerized images of 52 regular playing cards displayed using MATLAB. Card faces were displayed for $1 \mathrm{~s}$, after which the card back was displayed for $2 \mathrm{~s}$ plus a variable jitter between 0.1 and $1 \mathrm{~s}$ (M: $0.5 \mathrm{~s}, \mathrm{SD}: 0.24 \mathrm{~s})$. Using a 4 button box in their right hand, volunteers were instructed to press button 1 for HEART cards, button 2 for SPADES ('sort' trials), and make no response to CLUBS or DIAMONDS ('withhold' trials). Volunteers were instructed to respond as quickly as possible. The ongoing task was practised at each of the three sessions. In both scanning sessions, volunteers practised the ongoing sort task before entering the scanner, and were then additionally given the PM instruction: they were asked to respond by pressing button 3 for any occurrence of a number '7' card independent of suit (the PM intention). Thus, when in the scanner, participants performed the ongoing task as practised, but with the additional PM instruction. Within each scanner session, each volunteer sorted eight decks of cards with the PM intention over a total period of $\sim 15 \mathrm{~min}$. Thus, there were a total of 416 trials, of which 32 were PM trials, 192 were 'sort' trials, and 192 were 'withhold' trials. Card order was pseudo-randomized, and permuted to maximize estimability of each event type (Friston et al, 1999) while ensuring a delayed onset and minimum separation of PM events. Specifically, the constraints were that PM cards never occurred in the first seven cards of the entire sequence, and that there were always at least three intervening cards between PM events. Since each deck remained independent of the next, we also required that one of the 4 PM trials per deck occurred within each quarter (13 cards). Accuracy and reaction times to Sort and PM trials were recorded.

\section{fMRI Recording and Analysis}

T2*-weighted images were acquired at $1.5 \mathrm{~T}$ (Siemens Avanto). To minimize signal artifacts originating from the sinuses, axial slices were tilted $30^{\circ}$ from inter-commissural plane. Thirty-six $3 \mathrm{~mm}$ slices $(0.75 \mathrm{~mm}$ interslice gap) were acquired with an in-plane resolution of $3 \mathrm{~mm} \times 3 \mathrm{~mm}$ ( $\mathrm{TR}=3300 \mathrm{~ms}$ per volume, $\mathrm{TE}=50 \mathrm{~ms}$ ). Images were preprocessed using SPM8 (http://www.fil.ion.ucl.ac.uk/ $\mathrm{spm} /$ ). Raw images were spatially realigned and unwarped, spatially normalized to standard space and smoothed $(8 \mathrm{~mm}$ kernel). fMRI data were analyzed with the standard hierarchal model approach employed in SPM. For each subject, three trial types (sort, withhold, and PM) were modeled in first level design matrices (each session was analyzed in a separate design matrix) which also included six movement parameters estimated during preprocessing. For Sort and PM trials, we only included trials where correct responses were made (first button response only). In a separate analysis, the reaction time for each Sort and PM trial was included as a parametric modulator, and modeled as a separate column in the design matrix. Drug (nicotine/ placebo), genotype, and condition (sort, withhold, and PM and, in the reaction time analysis, sort/PM modulated by reaction time) were then entered into a second level full factorial model for the estimation of group effects. Gender was entered as a covariate. Main effects of nicotine are reported at $P<0.05$ FWE-corrected, following the application of a mask that included bilateral precuneus, inferior parietal, and extrastriate regions, since previous work has consistently shown nicotine effects in these regions during $\mathrm{PM}$ and other attentionally demanding tasks (Lawrence et al, 2002; Rusted et al, 2011; Thiel et al, 2005). All other analyses are presented at a threshold of $P<0.05$ FWEcorrected. Small volume corrections (where appropriate, based on the APOE and PM literature) are also reported at $P<0.05$ FWE-corrected. A priori regions of interest included areas most consistently implicated in PM (rostral prefrontal cortex (BA10 and BA11/12), dorsal anterior cingulate (BA32) and inferior parietal lobe (BA40)) and areas where differences between $\varepsilon 3$ and $\varepsilon 4$ have been reliably demonstrated (hippocampal formation). Regions of interest were defined using the Wake Forest University PickAtlas. Anatomical localization of clusters was performed using the Talairach Daemon (University of Texas, USA), and the anatomy toolbox for SPM (Eickhoff et al, 2005). 


\section{RESULTS}

\section{Behavioral Data}

Accuracy and RT data were analyzed using $2 \times 2 \times 2$ mixed factorial ANOVAs. Drug (placebo/nicotine) was entered as a within-subjects variable, session order (nicotine first or second), genotype (APOE 3/APOE 4), and gender were entered as between-subject variables. Significance was assessed at $P<0.05$. For summary data, see Table 2 .

Sort trials. No significant main effects of genotype, drug, session, or gender were found on sort accuracy, and no significant interactions were found. On reaction times, there were no significant main effects of genotype, drug, or session, and no significant interactions were found.

PM trials. No significant effects of genotype or drug were found on PM accuracy, and no significant interactions. There was a main effect of gender $\left(\mathrm{F}_{(1,35)}=4.90, P=0.03\right)$, with females being more accurate, but no interactions were found. For PM reaction times, there was no significant main effects of drug, genotype, or gender, but there was a significant drug $\times$ genotype interaction $\left(\mathrm{F}_{(1,35)}=5.26\right.$, $P=0.03)$. Analyzing data separately for each genotype showed that nicotine significantly reduced reaction times in $\varepsilon 4 \mathrm{~s}(t(19)=2.14, \quad P=0.045$ two-tailed) but not in $\varepsilon 3 \mathrm{~s}$ $(t(19)<1)$. Independent samples $t$-tests following placebo revealed no significant effect of genotype on accuracy or reaction times $(t(38)=1.356, P=0.18$ two-tailed test). This analysis was between subjects however, and consequently had less statistical power than the within-subjects analyses described above. There was a significant main effect of session $(\mathrm{F}(1,36)=49.39, \quad P \leqslant 0.001)$ and a significant drug $\times$ session order effect $(F(1,36)=19.31$, $P=0.001)$. Reductions in reaction time under nicotine were more pronounced when nicotine was presented in the second imaging session. There was no significant drug $\times$ genotype $\times$ session effect.

\section{fMRI Data}

Main effect of genotype. There were no significant main effects of genotype.

Main effect of nicotine in parietal lobe. A main positive effect of nicotine was identified in right precuneus $(x=8$, $y=-44, z=45 ; k=77 ; P=0.045$ FWE-corrected) and right inferior parietal lobule (IPL) $(x=58, y=-46, z=28$; $k=72 ; P=0.031 \mathrm{FWE}$-corrected), see Figure 1a. There were no areas where activity was decreased under nicotine.

Genotype-by-drug interactions. There was no significant genotype-by-drug interaction. However, the behavioral data showed that nicotine effects were restricted to PM trials in $\varepsilon 4$ carriers only. Applying the t-contrast $\varepsilon 4$ PM Nicotine $>$ Placebo revealed activation in extrastriate visual regions (BA18) $(x=6, y=-84, z=24 ; k=97 ; P=0.01 \quad$ FWEcorrected after SVC for cuneus), see Figure 1b. In this region, activity was significantly different between genotypes on PM trials under placebo $(x=6, y=-82, z=22$; $k=84 ; P=0.024$ FWE-corrected after SVC for cuneus, using the t-contrast $\varepsilon 4 \mathrm{PM}$ placebo $<\varepsilon 3 \mathrm{PM}$ placebo). Applying the $\mathrm{t}$-contrast $\& 4 \mathrm{PM}$ Placebo $>$ Nicotine revealed no significant activation.

Genotype-by-trial type interaction in medial BA10. An interaction of genotype $(\varepsilon 3, \varepsilon 4)$ by trial type (sort, withhold, PM) was identified in medial BA10 ( $x=-6, y=54, z=14$; $k=27 ; P=0.004 \mathrm{FWE}$-corrected); see Figure 1c. In BA10, $\varepsilon 4$ carriers showed decreases in $\mathrm{PM}$ activity relative to $\varepsilon 3$ carriers $(P=0.031$ FWE-small volume corrected).

Genotype-by-trial type-by-drug interaction in right hippocampus. A 3-way interaction between genotype $(\varepsilon 3, \varepsilon 4)$, trial type (sort, withhold, $\mathrm{PM}$ ) and drug (nicotine, placebo) identified the right hippocampus (rHipp, $x=32, y=-18$, $z=-28, k=18, P=0.031$ FWE corrected after SVC for right hippocampal formation), see Figure 1d. Peak activation was localized to the subiculum. We explored this interaction using the $\mathrm{t}$-contrast (Nicotine $\mathrm{PM}>$ Placebo $\mathrm{PM})$. In $\varepsilon 4$ carriers, this contrast was significant at $P=0.038$ (FWE-corrected) after small volume correction while in $\varepsilon 3$ carriers no significant activity was found.

Parametric modulation by reaction time (PM trials). A significant main effect of modulation by PM reaction time was observed in left middle cingulate cortex $(x=-6$, $y=-42, z=+50, k=5, P=0.012$ FWE-corrected), and left inferior parietal $(x=-38, y=-32, z=44, k=31$, $P=0.006$ FWE-corrected). In medial BA10, two distinct loci of activation were observed, area $1(x=-10, y=62, z=12$, $k=6, P=0.015$ FWE-corrected) and area $2(x=-4, y=62$, $z=4, k=10, P=0.012 \mathrm{FWE}$-corrected). Figure 1e shows the BA10 activations and associated contrast estimates, which

Table 2 Accuracy Scores and Reaction Times (in ms) for Ongoing and PM Trials

\begin{tabular}{|c|c|c|c|c|c|}
\hline \multirow[t]{2}{*}{ Genotype } & \multirow[t]{2}{*}{ Drug } & \multicolumn{2}{|c|}{ Ongoing trials } & \multicolumn{2}{|c|}{ PM trials } \\
\hline & & $\%$ Correct (SD) & RT (SD) & $\%$ Correct (SD) & RT (SD) \\
\hline & Placebo & $94.1(4.0)$ & $613.1(19.1)$ & $83.4(11.2)$ & $674.4(17.6)$ \\
\hline APOE4 & Nicotine & $94.5(3.6)$ & $608.1(22.0)$ & $78.0(\mid 4.5)$ & $678.9 *(17.8)$ \\
\hline
\end{tabular}

*Significantly different to APOE4 placebo (at $P<0.05$ ). 


\section{a Main effect of nicotine}

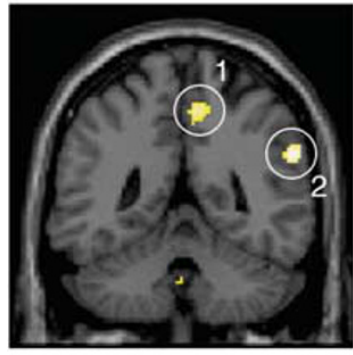

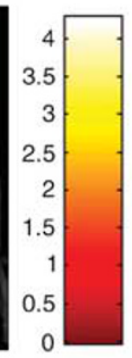

1

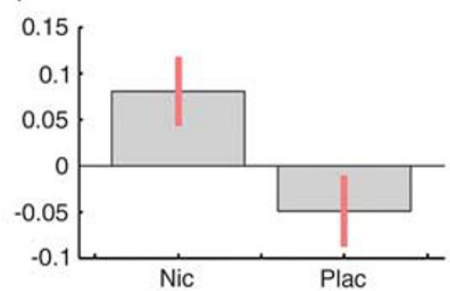

2

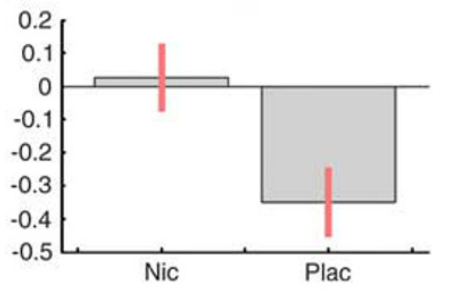

b

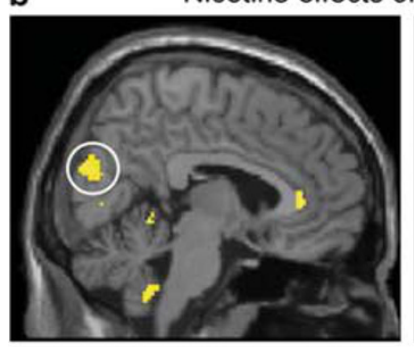

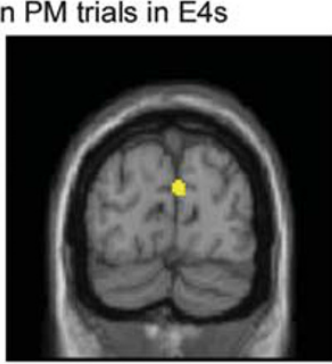

C

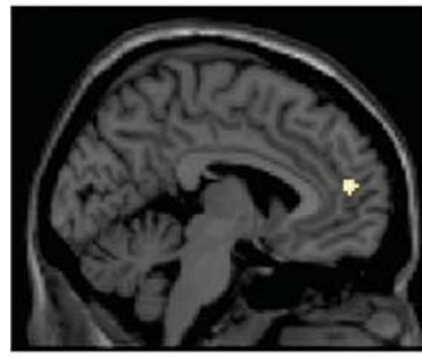

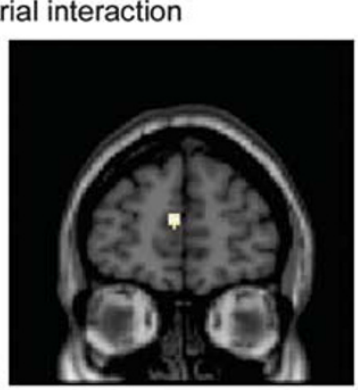

d

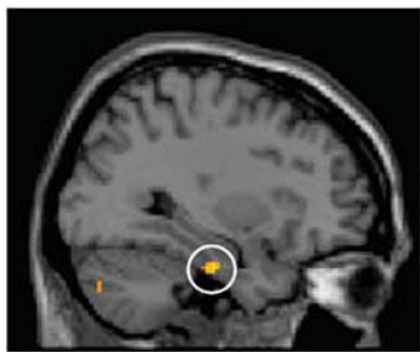

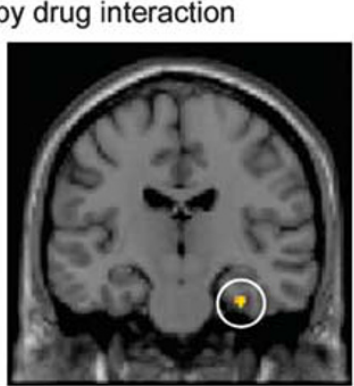
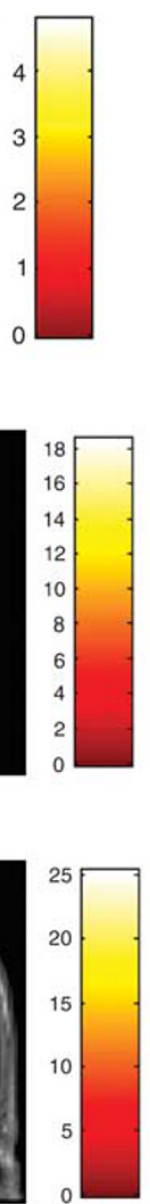

e Modulation by PM reaction time

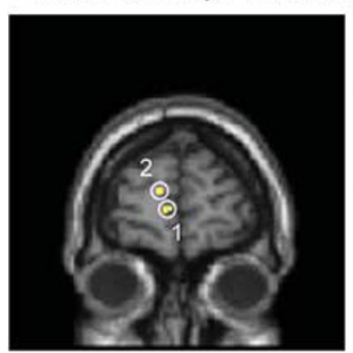

1

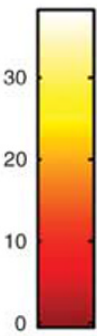

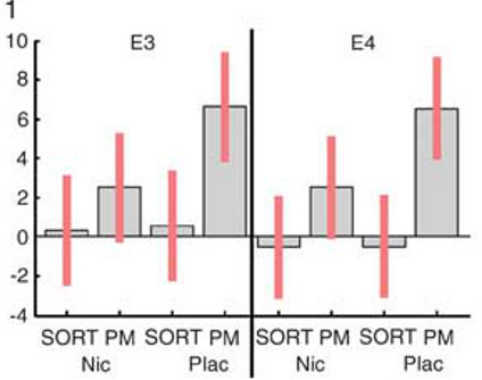
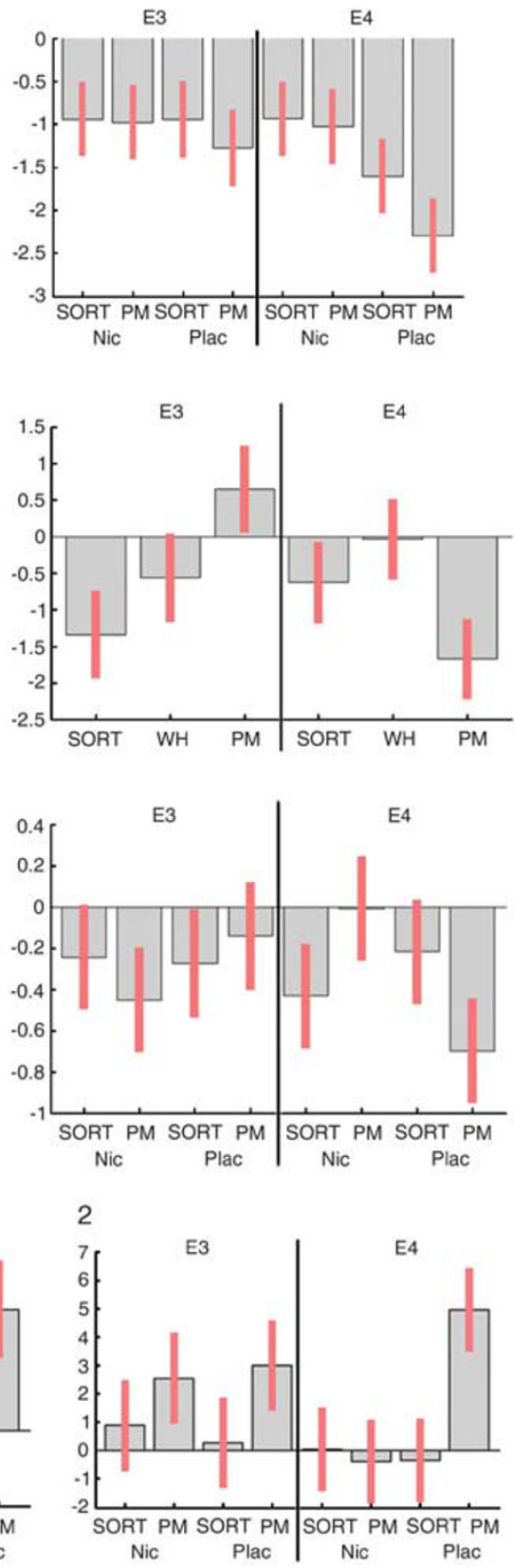

Figure I (a) Main positive effect of nicotine (across all trials and participants) in right precuneus (I) and right inferior parietal lobule (2), and associated parameter estimates with $90 \%$ confidence intervals. (b) On PM trials only, activity in extrastriate cortex was greater in $\varepsilon 4$ carriers under nicotine. (c) Genotype-by-trial type interaction in medial BAIO and associated parameter estimates for SORT trials (sort for spades/hearts), WH trials (withhold response, to hearts/clubs) and PM trials ('7' card from any suit). (d) Interaction between genotype $(\varepsilon 3, \varepsilon 4)$, trial type (SORT, PM), and drug (NIC, PLAC) in right hippocampus and associated mean parameter estimates (for brevity, WH trials are not shown, since estimates did not deviate from zero across drug/ genotype). (e) Activity modulated by PM reaction time in two subregions of medial BAIO (I) and (2). Contrast estimates for (I) and (2) showing different activation patterns for $\varepsilon 4$ carriers under nicotine. 
represent the strength of the correlation between activity and reaction time. These contrast estimates suggest a difference between the BA10 regions in terms of activation patterns. In area $1(-4,62,-4)$, activity correlated with $\mathrm{PM}$ reaction time under all conditions. In area $2(-10,62$, 12 ), this relationship was also present but appears weaker in $\varepsilon 4$ carriers under nicotine (see Figure 1e). This was confirmed using the t-contrast (Nic \&4 PM $<$ Plac $\varepsilon 4 \mathrm{PM}$ ). After small volume correction for BA10, this activity was significant $(k=28, P=0.042 \mathrm{FWE}$-corrected) in area 2 , but was not present in area 1.

\section{DISCUSSION}

On a PM task, prior administration of the cognitive enhancer nicotine reduced reaction times to respond to a PM cue, but only in $\varepsilon 4$ carriers. This accords with previous work employing the same PM paradigm and similar nicotine doses (Marchant et al, 2010), suggesting that nicotine selectively benefits $\varepsilon 4$ carriers on this task.

Unlike that previous study (which was a behavioral study only), our $\varepsilon 4$ volunteers did not record better performance than their $\varepsilon 3$ peers on the task overall. In common with that study, however, they did show a nicotine advantage for PM responses (here the advantage was observed in reaction time, previously it was observed in accuracy). They did not show nicotinic enhancement on the ongoing card sort task, perhaps because accuracy levels were at ceiling (mean accuracy approaching 95\%). These differences are likely a consequence of the extended number of trials associated with the fMRI event-based design. In the current study, participants practised card sorting before each of the three sessions, and they sorted eight decks in each fMRI session, compared with two decks in a single session in the Marchant study. In addition, the Marchant study involved a longer cognitive battery immediately before the card sort task, raising the possibility that resistance to fatigue could contribute to $\varepsilon 4$ performance advantages. This needs to be explored. Nevertheless, both the current and previous studies support the notion that $\varepsilon 4$ carriers may be more sensitive to the effects of nicotine.

The fMRI data identified differences in the way $\varepsilon 3$ and $\varepsilon 4$ carriers activated task-relevant regions. APOE status interacted significantly with trial type (sort/withhold/PM) in a rostral subregion of medial BA10. Specifically, $\varepsilon 4$ carriers showed medial BA10 deactivation in response to PM trials whereas $\varepsilon 3$ carriers deactivated only during the ongoing task. This pattern was independent of nicotine delivery. In healthy young adults, imaging studies have shown (typically using blocked designs) that BA10 deactivates in response to PM tasks (Burgess et al, 2001, 2003; Simons et al, 2006). Our previous work with the same PM task employed here also found medial BA10 deactivations in response to PM trials (Rusted et al, 2011). Since functional deactivation of BA10 is frequently observed during cognitive tasks, it has been argued that this may reflect an attentional shift (Burgess et al, 2007). Conversely, increased activity within this region is typically observed while subjects are at rest, and consequently medial prefrontal activity is considered a prominent feature of DMN activity (Greicius et al, 2003). The BA10 deactivations we observed could therefore reflect a downregulation of DMN activity (Mason et al, 2007). In the resting state, $\varepsilon 4$ carriers show enhanced co-activations between medial prefrontal cortex and other regions within the DMN (Filippini et al, 2009; Fleisher et al, 2009; Sheline et al, 2010). In sum, the data are consistent with the notion of genotype differences in functional responsivity within medial BA10, with deactivation patterns suggesting greater suppression of resting state by $\varepsilon 4$ carriers in response to the PM component of the task.

In addition, the fMRI data indicated that two distinct caudal BA10 subregions showed a relationship with reaction times to PM targets, in keeping with the notion that BA10 is a critical structure in PM task performance (see parameter estimates in Figure 1e). Thus, the event-related design used here has provided important data directly linking medial BA10 deactivations to behavior on PM trials. Other imaging studies have consistently pointed to medial BA10 involvement, but the exact locus of activity varies between studies (Burgess et al, 2011). Data provided here suggest that subregions of medial BA10 may be sensitive to different aspects of PM task requirements. Although both subregions showed significant modulation by reaction time, one subregion showed modulation on all PM trials, while in the other (located more dorsally) modulation was absent in $\varepsilon 4$ carriers under nicotine (see Figure 1e). We suggest that the performance-enhancing effects of nicotine in $\varepsilon 4$ carriers might be related to this finding. In these individuals, nicotine seems to disrupt reaction time modulation of activity in the more dorsal region, implying a different functional role under nicotine. Thus, altered processing within this subregion could be a factor in the observed behavioral advantages. Although further work is clearly needed, we have shown for the first time that subregions of medial BA10 that can be directly related to PM reaction time are differentially modulated by APOE genotype, and by nicotine.

Behaviorally, a drug-by-genotype interaction was found in reaction times to the PM target, with nicotine significantly reducing reaction times in $\varepsilon 4$ carriers only. This interaction was not significant in the fMRI data, but applying a t-contrast to explore the neural correlates of this effect in $\varepsilon 4$ carriers on PM trials, we identified significant activations in extrastriate visual areas. Activity in this region was enhanced by nicotine, whereas no effects were evident in 83 carriers. Some previous studies have shown increased activity in extrastriate visual areas under nicotine (Lawrence et al, 2002; Tanabe et al, 2011) and this is thought to reflect enhanced processing of external visual stimuli. We found that only $\varepsilon 4$ carriers demonstrate such a pattern of activity under nicotine. This accords with the behavioral data, and suggests that nicotine might improve performance in $\varepsilon 4$ carriers by amplifying the representation of external stimuli, facilitating selection of the appropriate behavioral response, leading to faster reaction times. The amplification of stimuli representations could be interpreted in terms of enhanced selective attention, since selective attention has been consistently shown to modulate extrastriate activity (Chen et al, 2012; Nobre et al, 1998). It should be noted that extrastriate activity itself was not found to be modulated by reaction time, but it is likely that many other intervening factors are involved as activity propagates upstream through the task-relevant network. 
Thus, one might not necessarily expect a straightforward relationship between activity relating to visual processing of the cue and the speed of response. Nevertheless, further work is needed to link nicotine-induced behavioral enhancements in $\varepsilon 4$ carriers to extrastriate activity.

Independent of genotype, our data indicate an influence of acutely administered nicotine on right precuneus and inferior parietal response. The localization of nicotinic modulation of neural activity to right IPL replicates our preliminary findings with this task (Rusted et al, 2011). Fronto-parietal activation is generally found during PM tasks (Gilbert et al, 2009; Martin et al, 2007). The parietal contribution likely encapsulates the representation of competing responses (Bunge et al, 2002) as well as the allocation of attentional resources (Bisley and Goldberg, 2010), whereas frontal activity may more specifically reflect $\mathrm{PM}$ response.

Nicotine has been shown to reduce parietal activity associated with attentional reorienting during a covert attention task (Thiel et al, 2005). In the resting state, nicotine has been shown to decrease activity in certain regions of the DMN, including the precuneus (Tanabe et al, 2011). This has led to suggestions that nicotine effects might be partly due to a disengagement from resting brain function (Hahn et al, 2007). Whereas reduced parietal activity had been reported previously with the PM task used here (Rusted et al, 2011), in the present study we observed nicotine-related enhancements in the right IPL and precuneus activity across all trial types regardless of genotype. In common with some other studies (Ernst et al, 2001; Ghatan et al, 1998), in 83 carriers this increased activation occurred in the absence of any behavioral effects of nicotine. Such inconsistencies are difficult to explain but are typical of the published nicotine literature, with reports of both increased (Ernst et al, 2001; Lawrence et al, 2002) and decreased (Thiel and Fink, 2008; Thiel et al, 2005) parietal activity in response to nicotine. Large individual differences in the neural and behavioral response to nicotine (Giessing et al, 2007) as well as differences in the doses of nicotine used across studies may contribute to these inconsistent findings, particularly when sample sizes are small.

Though the increased activation pattern was independent of genotype, the fMRI data reported here are suggestive of some differences between genotypes in the response to task demands. In comparison with the $\varepsilon 3$ carriers, $\varepsilon 4$ carriers showed a tendency toward greater neural activation under nicotine. This may indicate genotype differences in the deployment of top-down $v s$ bottom-up processing in response to task demands. A similar argument was proposed by Furey et al (2000) to explain changes in activation patterns between visual cortex and frontal regions in response to cholinergic stimulation in healthy young adults. As mentioned previously, the $\varepsilon 4$ group in the present study showed nicotine-induced activations in extrastriate regions. A role for acetylcholine in influencing both top-down and bottom-up attentional processes is widely accepted (Bentley et al, 2011; Sarter et al, 2006; Yu and Dayan, 2005). It is possible genotype differences in cholinergic tone and sensitivity to cholinergic manipulation may provide a parsimonious explanation of the pattern of neural and behavioral outcomes that we have observed, and this is a hypothesis that warrants further development. Interactions between the $\varepsilon 4$ allele and cholinergic activity have been discussed in relation to older adults (Greenwood et al, 2005a, 2005b; Parasuraman et al, 2002), but differences in a younger population have not been systematically explored.

We observed also an interaction of drug, trial type, and genotype within the right hippocampus. Specifically, under nicotine, $\varepsilon 4$ carriers showed a relative activation in this region during PM trials. A previous study has reported nicotine effects in the hippocampal formation at rest (Kumari and Gray, 2003). While we found nicotine effects to be localized to the precuneus and IPL, altered connectivity between precuneus and hippocampus has been reported in $\varepsilon 4$ carriers (Sheline et al, 2010) and this might underlie the enhanced hippocampal activation observed here for $\varepsilon 4$ carriers under nicotine. Hippocampal activity has been implicated in PM performance, likely reflecting episodic activation of the intention (Martin et al, 2007). Furthermore, patients with MTL damage show PM impairments (Adda and Castro, 2008). It is possible therefore that nicotine-induced MTL activation in 84 carriers might be beneficial to PM. The PM task employed here, however, does not tend to elicit hippocampal activity (Rusted et al, 2011) and, in the present study, we found no relationship between the observed hippocampal activity and PM reaction times, arguing against its contribution to PM task performance. Indeed, $\varepsilon 4$ carriers have been shown to activate the MTL even when superfluous to task requirements (Trachtenberg et al, 2012a), and MTL overactivity in $\varepsilon 4$ carriers is frequently reported during episodic memory tasks (Bookheimer et al, 2000; Dennis et al, 2010). If, as has been suggested, enhanced MTL recruitment reflects some sort of compensatory mechanism in $\varepsilon 4$ carriers (Bondi et al, 2005), the data presented here would suggest that such a mechanism is potentiated by nicotine, although this compensatory activity may be independent of any actual behavioral benefits. Further work is needed to determine the precise conditions under which this mechanism is activated, and whether it might contribute to the cognitive performance advantages sometimes observed in young $\varepsilon 4$ carriers.

In summary, we have shown that nicotine improves reaction times to PM trials, but only in $\varepsilon 4$ carriers. This suggests that $\varepsilon 4$ carriers may exhibit greater sensitivity to nicotine and that this may be exploited to improve cognitive performance. We have further shown that PM-related activity in medial BA10 differentiated $\varepsilon 3$ and $\varepsilon 4$ carriers. A rostral subregion of BA10 showed a genotype-specific dissociation in activity, while two other subregions (located more caudally) showed activity modulated by reaction time to PM trials. Nicotine in 84 carriers abolished this modulation in the more dorsal region. These results confirm that medial BA10 deactivations are closely tied to PM performance and suggest drug and genotype specificity within various subregions of this structure.

Nicotine enhanced parietal activation across all participants, but genotype-specific effects were observed in extrastriate cortex. Nicotine enhanced activity in this region just in $\varepsilon 4$ carriers, suggesting that nicotine may promote faster PM responses in $\varepsilon 4$ carriers by amplifying stimulus representations and facilitating bottom-up processing. 
In line with other studies showing hippocampal overactivity in $\varepsilon 4$ carriers, we found that uniquely $\varepsilon 4$ carriers activate right hippocampus under nicotine, although this activity did not appear to relate to task performance. Further research specifically focused on the integration of medial prefrontal, precuneus/inferior parietal and hippocampal activity during PM may allow additional insight into how neural activity and cholinergic system modulation of behavior is influenced by APOE allele status.

\section{ACKNOWLEDGEMENTS}

This study was funded by a BBSRC grant to Jenny Rusted (BB/H000518/1). The funders had no role in study design, data collection and analysis, decision to publish, or preparation of the manuscript. We thank McNeil $A B$, Helsingborg, Sweden for providing the nasal sprays. We thank John Broulidakis for assistance with the behavioral data analysis, and three anonymous reviewers for their extremely useful suggestions regarding the analytical approaches and interpretation.

\section{DISCLOSURE}

NT has received research grants (unrelated to this study) from Pfizer and Novartis. He also received consultation and/or speaker fees from Pfizer, Eli Lilly, AstraZeneca, Shire, Novartis, and Lundbeck.

\section{REFERENCES}

Adda CC, Castro LH, Alem-Mar e Silva LC, de Manreza ML, Kashiara R (2008). Prospective memory and mesial temporal epilepsy associated with hippocampal sclerosis. Neuropsychologia 46: 1954-1964.

Arendt T, Schindler C, Bruckner MK, Eschrich K, Bigl V, Zedlick D et al (1997). Plastic neuronal remodeling is impaired in patients with Alzheimer's disease carrying apolipoprotein epsilon 4 allele. J Neurosci 17: 516-529.

Bentley P, Driver J, Dolan RJ (2011). Cholinergic modulation of cognition: insights from human pharmacological functional neuroimaging. Prog Neurobiol 94: 360-388.

Bisley JW, Goldberg ME (2010). Attention, intention, and priority in the parietal lobe. Annu Rev Neurosci 33: 1-21.

Bloss CS, Delis DC, Salmon DP, Bondi MW (2010). APOE genotype is associated with left-handedness and visuospatial skills in children. Neurobiol Aging 31: 787-795.

Bondi MW, Houston WS, Eyler LT, Brown GG (2005). fMRI evidence of compensatory mechanisms in older adults at genetic risk for Alzheimer disease. Neurology 64: 501-508.

Bookheimer SY, Strojwas MH, Cohen MS, Saunders AM, PericakVance MA, Mazziotta JC et al (2000). Patterns of brain activation in people at risk for Alzheimer's disease. $N$ Engl J Med 343: 450-456.

Borghesani PR, Johnson LC, Shelton AL, Peskind ER, Aylward EH, Schellenberg GD et al (2008). Altered medial temporal lobe responses during visuospatial encoding in healthy $\mathrm{APOE}^{\star} 4$ carriers. Neurobiol Aging 29: 981-991.

Braak H, Braak E (1997). Diagnostic criteria for neuropathologic assessment of Alzheimer's disease. Neurobiol Aging 18(4 Suppl): S85-S88.

Bunge SA, Hazeltine E, Scanlon MD, Rosen AC, Gabrieli JD (2002). Dissociable contributions of prefrontal and parietal cortices to response selection. Neuroimage 17: 1562-1571.
Burgess PW, Gilbert SJ, Dumontheil I (2007). Function and localization within rostral prefrontal cortex (area 10). Philos Trans R Soc Lond B Biol Sci 362: 887-899.

Burgess PW, Gonen-Yaacovi G, Volle E (2011). Functional neuroimaging studies of prospective memory: what have we learnt so far? Neuropsychologia 49: 2246-2257.

Burgess PW, Quayle A, Frith CD (2001). Brain regions involved in prospective memory as determined by positron emission tomography. Neuropsychologia 39: 545-555.

Burgess PW, Scott SK, Frith CD (2003). The role of the rostral frontal cortex (area 10) in prospective memory: a lateral versus medial dissociation. Neuropsychologia 41: 906-918.

Chen AJ, Britton M, Turner GR, Vytlacil J, Thompson TW, D'Esposito M (2012). Goal-directed attention alters the tuning of object-based representations in extrastriate cortex. Front Hum Neurosci 6: 187.

Deary IJ, Whiteman MC, Pattie A, Starr JM, Hayward C, Wright AF et al (2002). Cognitive change and the APOE epsilon 4 allele. Nature 418: 932.

Dennis NA, Browndyke JN, Stokes J, Need A, Burke JR, Welsh-Bohmer KA et al (2010). Temporal lobe functional activity and connectivity in young adult APOE varepsilon4 carriers. Alzheimers Dement 6: 303-311.

Driscoll I, McDaniel MA, Guynn MJ (2005). Apolipoprotein E and prospective memory in normally aging adults. Neuropsychology 19: $28-34$.

Eickhoff SB, Stephan KE, Mohlberg H, Grefkes C, Fink GR, Amunts $\mathrm{K}$ et al (2005). A new SPM toolbox for combining probabilistic cytoarchitectonic maps and functional imaging data. Neuroimage 25: 1325-1335.

Ernst M, Matochik JA, Heishman SJ, Van Horn JD, Jons PH, Henningfield JE et al (2001). Effect of nicotine on brain activation during performance of a working memory task. Proc Natl Acad Sci USA 98: 4728-4733.

Filippini N, Ebmeier KP, MacIntosh BJ, Trachtenberg AJ, Frisoni GB, Wilcock GK et al (2011). Differential effects of the APOE genotype on brain function across the lifespan. Neuroimage 54: 602-610.

Filippini N, MacIntosh BJ, Hough MG, Goodwin GM, Frisoni GB, Smith SM et al (2009). Distinct patterns of brain activity in young carriers of the APOE-epsilon4 allele. Proc Natl Acad Sci USA 106: 7209-7214.

Fleisher AS, Sherzai A, Taylor C, Langbaum JB, Chen K, Buxton RB (2009). Resting-state BOLD networks versus task-associated functional MRI for distinguishing Alzheimer's disease risk groups. Neuroimage 47: 1678-1690.

Friston KJ, Zarahn E, Josephs O, Henson RN, Dale AM (1999). Stochastic designs in event-related fMRI. Neuroimage 10: 607-619.

Furey ML, Pietrini P, Haxby JV (2000). Cholinergic enhancement and increased selectivity of perceptual processing during working memory. Science 290: 2315-2319.

Ghatan PH, Ingvar M, Eriksson L, Stone-Elander S, Serrander M, Ekberg $\mathrm{K}$ et al (1998). Cerebral effects of nicotine during cognition in smokers and non-smokers. Psychopharmacology (Berl) 136: 179-189.

Giessing C, Fink GR, Rosler F, Thiel CM (2007). fMRI data predict individual differences of behavioral effects of nicotine: a partial least square analysis. J Cogn Neurosci 19: 658-670.

Gilbert SJ, Gollwitzer PM, Cohen AL, Burgess PW, Oettingen G (2009). Separable brain systems supporting cued versus self-initiated realization of delayed intentions. J Exp Psychol Learn Mem Cogn 35: 905-915.

Greenwood PM, Lambert C, Sunderland T, Parasuraman R (2005a). Effects of apolipoprotein E genotype on spatial attention, working memory, and their interaction in healthy, middle-aged adults: results From the National Institute of Mental Health's BIOCARD study. Neuropsychology 19: 199-211. 
Greenwood PM, Sunderland T, Putnam K, Levy J, Parasuraman R (2005b). Scaling of visuospatial attention undergoes differential longitudinal change as a function of APOE genotype prior to old age: results from the NIMH BIOCARD study. Neuropsychology 19: $830-840$

Greicius MD, Krasnow B, Reiss AL, Menon V (2003). Functional connectivity in the resting brain: a network analysis of the default mode hypothesis. Proc Natl Acad Sci USA 100: 253-258.

Hahn B, Ross TJ, Yang Y, Kim I, Huestis MA, Stein EA (2007). Nicotine enhances visuospatial attention by deactivating areas of the resting brain default network. J Neurosci 27: 3477-3489.

Han SD, Bondi MW (2008). Revision of the apolipoprotein E compensatory mechanism recruitment hypothesis. Alzheimers Dement 4: 251-254.

Han SD, Houston WS, Jak AJ, Eyler LT, Nagel BJ, Fleisher AS et al (2007). Verbal paired-associate learning by APOE genotype in non-demented older adults: fMRI evidence of a right hemispheric compensatory response. Neurobiol Aging 28: 238-247.

Hubacek JA, Pitha J, Skodova Z, Adamkova V, Lanska V, Poledne $\mathrm{R}$ (2001). A possible role of apolipoprotein $\mathrm{E}$ polymorphism in predisposition to higher education. Neuropsychobiology 43: 200-203.

Kumari V, Gray JA, ffytche DH, Mitterschiffthaler MT, Das M, Zachariah E et al (2003). Cognitive effects of nicotine in humans: an fMRI study. Neuroimage 19: 1002-1013.

Lawrence NS, Ross TJ, Stein EA (2002). Cognitive mechanisms of nicotine on visual attention. Neuron 36: 539-548.

Mahley RW (1988). Apolipoprotein E: cholesterol transport protein with expanding role in cell biology. Science 240: 622-630.

Marchant NL, King SL, Tabet N, Rusted JM (2010). Positive effects of cholinergic stimulation favor young APOE epsilon4 carriers. Neuropsychopharmacology 35: 1090-1096.

Marchant NL, Trawley S, Rusted JM (2008). Prospective memory or prospective attention: physiological and pharmacological support for an attentional model. Int J Neuropsychopharmacol 11: 401-411.

Martin T, McDaniel MA, Guynn MJ, Houck JM, Woodruff CC, Bish JP et al (2007). Brain regions and their dynamics in prospective memory retrieval: a MEG study. Int J Psychophysiol 64: 247-258.

Mason MF, Norton MI, Van Horn JD, Wegner DM, Grafton ST, Macrae CN (2007). Wandering minds: the default network and stimulus-independent thought. Science 315: 393-395.

Mondadori CR, de Quervain DJ, Buchmann A, Mustovic H, Wollmer MA, Schmidt CF et al (2007). Better memory and neural efficiency in young apolipoprotein E epsilon4 carriers. Cereb Cortex 17: 1934-1947.

Nelson HE (1982). The National Adult Reading Test (NART): Test Manual. NFER-Nelson: Windsor.

Nobre AC, Allison T, McCarthy G (1998). Modulation of human extrastriate visual processing by selective attention to colours and words. Brain 121, (Pt 7) 1357-1368.

Parasuraman R, Greenwood PM, Sunderland T (2002). The apolipoprotein E gene, attention, and brain function. Neuropsychology 16: 254-274.

Poirier J, Delisle MC, Quirion R, Aubert I, Farlow M, Lahiri D et al (1995). Apolipoprotein E4 allele as a predictor of cholinergic deficits and treatment outcome in Alzheimer disease. Proc Natl Acad Sci USA 92: 12260-12264.

Rocchi A, Pellegrini S, Siciliano G, Murri L (2003). Causative and susceptibility genes for Alzheimer's disease: a review. Brain Res Bull 61: 1-24.
Ruest T, Dowell NG, Evans SL, King SL, Tabet N, Tofts P (under review) MR imaging of carriers of the apolipoprotein $\mathrm{E}$ e4 allele - evidence for structural differences in normal appearing brain tissue in e4+ compared to e4- young adults.

Rusted J, Ruest T, Gray MA (2011). Acute effects of nicotine administration during prospective memory, an event related fMRI study. Neuropsychologia 49: 2362-2368.

Rusted JM, Sawyer R, Jones C, Trawley SL, Marchant NL (2009). Positive effects of nicotine on cognition: the deployment of attention for prospective memory. Psychopharmacology (Berl) 202: 93-102.

Rusted JM, Trawley S, Heath J, Kettle G, Walker H (2005). Nicotine improves memory for delayed intentions. Psychopharmacology (Berl) 182: 355-365.

Rusted JM, Warburton DM (1989). Effects of scopolamine on verbal memory; a retrieval or acquisition deficit? Neuropsychobiology 21: 76-83.

Sarter M, Gehring WJ, Kozak R (2006). More attention must be paid: The neurobiology of attentional effort. Brain Res Rev 51: $145-160$

Schneider NG, Lunell E, Olmstead RE, Fagerstrom KO (1996). Clinical pharmacokinetics of nasal nicotine delivery. A review and comparison to other nicotine systems. Clin Pharmacokinet 31: $65-80$.

Sheline YI, Morris JC, Snyder AZ, Price JL, Yan Z, D'Angelo G et al (2010). APOE4 allele disrupts resting state fMRI connectivity in the absence of amyloid plaques or decreased CSF Abeta42. J Neurosci 30: 17035-17040.

Simons JS, Scholvinck ML, Gilbert SJ, Frith CD, Burgess PW (2006). Differential components of prospective memory? Evidence from fMRI. Neuropsychologia 44: 1388-1397.

Tanabe J, Nyberg E, Martin LF, Martin J, Cordes D, Kronberg E et al (2011). Nicotine effects on default mode network during resting state. Psychopharmacology (Berl) 216: 287-295.

Thiel CM, Fink GR (2008). Effects of the cholinergic agonist nicotine on reorienting of visual spatial attention and top-down attentional control. Neuroscience 152: 381-390.

Thiel CM, Zilles K, Fink GR (2005). Nicotine modulates reorienting of visuospatial attention and neural activity in human parietal cortex. Neuropsychopharmacology 30: 810-820.

Trachtenberg AJ, Filippini N, Cheeseman J, Duff EP, Neville MJ, Ebmeier KP et al (2012a). The effects of APOE on brain activity do not simply reflect the risk of Alzheimer's disease. Neurobiol Aging 33: 618 e611-618 e613.

Trachtenberg AJ, Filippini N, Ebmeier KP, Smith SM, Karpe F, Mackay CE (2012b). The effects of APOE on the functional architecture of the resting brain. Neuroimage 59: 565-572.

Westlye ET, Lundervold A, Rootwelt H, Lundervold AJ, Westlye LT (2011). Increased hippocampal default mode synchronization during rest in middle-aged and elderly APOE epsilon4 carriers: relationships with memory performance. J Neurosci 31: 7775-7783.

Yu AJ, Dayan P (2005). Uncertainty, neuromodulation, and attention. Neuron 46: 681-692.

Yu YW, Lin CH, Chen SP, Hong CJ, Tsai SJ (2000). Intelligence and event-related potentials for young female human volunteer apolipoprotein E epsilon4 and non-epsilon4 carriers. Neurosci Lett 294: 179-181.

Zhou W, Xu D, Peng X, Zhang Q, Jia J, Crutcher KA (2008). Metaanalysis of APOE4 allele and outcome after traumatic brain injury. J Neurotrauma 25: 279-290. 